\title{
Low manganese and high iron levels in amniotic fluid correlate with fetal chromosomal abnormalities in pregnant women
}

\author{
J Suliburska ( $\square$ joanna.suliburska@up.poznan.pl ) \\ University of Life Sciences in Poznań \\ Jakub Pankiewicz \\ PreMediCare New Med
}

Adam Sajnóg

Adam Mickiewicz University in Poznań

Magdalena Paczkowska

Instytut Matki i Dziecka

\section{Beata Nowakowska}

Instytut Matki i Dziecka

Ewa Bakinowska

Poznań University of Technology

\section{Danuta Barałkiewicz}

Adam Mickiewicz University in Poznań

\section{Rafał Kocyłowski}

PreMediCare New Med

\section{Research Article}

Keywords: pregnancy, elements, amniotic fluid, chromosomal abnormalities

Posted Date: August 27th, 2021

DOI: https://doi.org/10.21203/rs.3.rs-841199/v1

License: (1) (1) This work is licensed under a Creative Commons Attribution 4.0 International License. Read Full License 


\section{Abstract}

This study aimed to check the association of essential and toxic elements in amniotic fluid (AF) with chromosomal abnormalities.

A total of 156 pregnant Polish white Caucasian women between the age of 20 and 43 years participated in the study. AF samples were collected during routine diagnostic and treatment procedures in pregnant women. Inductively coupled plasma mass spectrometry (ICP-MS) was used to determine the levels of various elements in AF. Genomic hybridization and cytogenic karyotype analysis were used. The results of the karyotype analysis indicated chromosomal abnormalities in 19 fetuses (over $12 \%$ of the total population) and it was mainly trisomy $21(\mathrm{~N}=11)$, trisomy $18(\mathrm{~N}=2)$, triploidy $(\mathrm{N}=2)$ and other chromosomal aberrations. It was found that a low concentration of manganese in AF was associated with chromosomal abnormalities in the foetus. High levels of iron and advanced age of the mother increased the risk of aneuploidy in the fetus. Principal component analysis (PCA) and Spearman correlation showed a strong correlation between essential and toxic elements in $\mathrm{AF}$, especially in groups with chromosomal abnormalities.

The results of this exploratory study indicate that the levels of essential and toxic elements in AF are associated with chromosomal abnormalities in the human fetus.

\section{Introduction}

Prenatal diagnostic procedures use amniotic fluid (AF) as material in search of chromosomal abnormalities, fetus anomalies and diseases. From the second trimester of pregnancy, fetal urine appears in AF. During early development, $A F$ is in contact with fetal skin, gastrointestinal tract and lungs. It can therefore be assumed that the composition of $\mathrm{AF}$ reflects the composition of fetal plasma ${ }^{1}$. The composition of AF depends on the mother's nutrition, including essential nutrients, and also on the mother's exposure to toxic substances ${ }^{2}$.

So far, few studies have described the relationship of mineral nutrition and the concentration of toxic elements in the mother's body, including amniotic fluid, with the risk of genetic and chromosomal abnormalities in the fetus and newborns. Because one of the most common chromosomal abnormalities in humans is Down's Syndrome (DS) which is genetically trisomy 21 , most of the results indicate the association between the concentration of elements and that disease. A meta-analysis showed that calcium, selenium and zinc levels in blood were lower in people with DS, while higher concentrations of zinc and copper were found in red cells in DS compared with the healthy group ${ }^{3}$. Altered iron metabolism and neuroinflammation was observed in DS and also in Alzheimer's disease ${ }^{4}$. Moreover, it was found that the prevalence of anaemia is higher in DS than in the general population ${ }^{5}$. In chromosomal abnormalities such as trisomy 18 and 21 , clinical symptoms include neurological disorders ${ }^{6,7}$. Some studies have suggested that overexpression of amyloid in the brain is the key element in the development of cognitive decline in people. It was found that extracellular accumulation of zinc and copper in amyloid and 
intracellular excess iron in neurons is associated with DS in humans, whereas in an animal study, in a mouse model of DS that calcium ion channel activity was observed to be higher than in the wild-type mouse $^{8}$. Some environmental factors may increase the risk of chromosomal aberration in pregnancy. It is known that metals such as $\mathrm{Cd}, \mathrm{Al}, \mathrm{As}, \mathrm{Pb}$ are neurotoxic and early exposure to these metals may cause neuronal developmental disorders ${ }^{9}$.

In our previous study, we indicated that some essential and toxic elements were associated with fetal congenital anatomical defects and growth disorders. We found that low concentrations of magnesium and vanadium in amniotic fluid and high concentrations of aluminium, cadmium and lead increased the risk of non-genetic birth defects ${ }^{10}$.

The influence of essential and toxic elements on maternal health and fetal development has not yet been fully investigated and the mechanisms are poorly understood. Recent research results indicate that mineral elements may be more important than assumed in assessing the risk of chromosomal abnormalities in the human fetus. Therefore, this exploratory analysis aimed to determine the association of selected essential and toxic elements in amniotic fluid with chromosomal disorders detected in fetuses of the population of white Caucasian mothers.

\section{Materials And Methods}

\subsection{Study design}

This was a retrospective cross-sectional study aiming to determine the levels of elements in the amniotic fluid of pregnant women classified as high risk for chromosomal abnormalities on the basis of firsttrimester screening tests. The AF was collected during a routine prenatal invasive procedure (amniocentesis) at 15-22 weeks of gestation performed between 2015 and 2020.

The study was conducted on 156 pregnant Polish women (white/Caucasian) who were under routine obstetric care. The women voluntarily participated in the study after signing an informed consent form. They consented to the secondary use of their samples. The study was conducted on patients qualified to participate based on inclusion and exclusion criteria.

The inclusion criteria were single viable intrauterine pregnancy in the second trimester and informed consent. The exclusion criteria were: multiple pregnancies, lack/withdrawal of the consent, too small amniotic fluid sample, excessive blood contamination in the amniotic fluid sample, maternal or intrauterine infection, fetal anatomic anomalies, maternal chronic illnesses, multidrug therapy.

A routine medical interview was conducted to collect data on maternity and pregnancy history of illnesses and operations, drugs and medications used. The week of gestation was determined by firsttrimester obstetric ultrasonography. Samples of amniotic fluid were collected from all women and genomic hybridization and cytogenic karyotype analysis were performed. According to the results of 
chromosome analysis, women were divided into two groups: the control group (normal karyotype) and the study group (with chromosomal abnormalities).

The study protocol was approved by the Bioethics Commission at Poznan University of Medical Sciences (approval no. 30/15) in Poland. This study was conducted in accordance with the Declaration of Helsinki. The study has been registered with ClinicalTrials.gov, ID: NCT03598361.

\subsection{Collection of biospecimens}

Amniotic fluid (AF) samples were obtained $(5 \mathrm{~mL})$ for genetic and element analysis following routine diagnostic amniocentesis at 15-22 weeks of gestation. Samples were collected by transabdominal aspiration using sterile 22-25G spinal and diagnostic puncture needles and plastic syringes free of organic and non-biological contamination. Each sample of fresh amniotic fluid was centrifuged for $5 \mathrm{~min}$ at $1300 \mathrm{rpm}$. Next, $2 \mathrm{ml}$ of supernatant was transferred to a new $2 \mathrm{ml}$ tube and frozen at $-80^{\circ} \mathrm{C}$ for analysis.

\subsection{Element measurements}

Measurement of essential and toxic elements in AF has been described by Markiewicz et al. ${ }^{11}$ and Kocylowski et al. ${ }^{10}$. This method involves the use of inductively coupled plasma mass spectrometry (ICP$\mathrm{MS}$ ) technique to assess the levels of $\mathrm{Li}, \mathrm{Mg}, \mathrm{Al}, \mathrm{Ca}, \mathrm{Cr}, \mathrm{Mn}, \mathrm{Fe}, \mathrm{Co}, \mathrm{Ni}, \mathrm{Cu}, \mathrm{As}$, Se, Sr and Cd in AF. Samples were mineralized in a high-pressure, closed microwave digestion system (Ethos One, Milestone). An Elan DRC II ICP-MS (PerkinElmer SCIEX, Ontario, Canada) was used to determine the content of elements.

Calibration based on a weighted least squares calibration curve was employed for all elements. Linearity - calculated as $R^{2}$ - was acceptable for all analyzed elements $\left(R^{2}>0.999\right)$. The trueness of the analytical method was assessed by analyzing the certified reference material (CRM) - Seronorm ${ }^{\text {'m }}$ Trace Elements Serum L-2. The values of recovery were within an acceptable range for all analytes, which demonstrates that the described analytical procedure is fit for the intended purpose.

\subsection{Genomic hybridization and cytogenic karyotype analysis}

\section{DNA Isolation}

Genomic DNA for CMA (chromosomal microarray analysis) was extracted from fresh amniotic fluid (AF) using the Sherlock kit (A\&A Biotechnology, Poland) according to the manufacturer's recommendations.

\section{Array design}


Array Comparative Genomic Hybridization (aCGH) was performed using 60K microarrays 8x60K from Oxford Gene Technology (CytoSure ISCA, v3). The array contains 51,317-mer oligonucleotide probes covering the whole genome with an average spatial resolution of $60 \mathrm{~Kb}$.

\section{Array Comparative Genomic Hybridization analysis}

Procedures for DNA denaturation, labelling, hybridization and washing were performed according to the manufacturer's instructions. Genomic DNA was labelled using a CytoSure Labelling Kit (Oxford Gene Technology), with no enzyme digestion. Hybridization was performed between 24 and 48 hours at $65^{\circ} \mathrm{C}$ in a rotator oven (Agilent). Arrays were washed using Agilent wash buffer 1 and 2, scanned using Agilent Technologies microarray scanner and signal intensities were measured using Feature Extraction software (Agilent Technologies). Agilent Feature Extraction software (V10.0) was used to quantify all scanned images.

CytoSure Interpret Software (Oxford Gene Technology) was used to perform data analysis based on the reference genome (NCBI37/hg19).

\section{Cytogenetic karyotype}

In most cases, part of the amniotic fluid sent for aCGH was also used for cytogenetic karyotype analysis.

Amniocytes were cultured using in situ vessels. Colony growth and mitotic activity were daily controlled after the 8th day of culture. Metaphase chromosomes were prepared according to standard procedures of GTG banding.

\subsection{Statistical analysis}

All statistical analyzes were performed with Statistica 13 for Windows and RStudio software (R version 3.4.0) R Core Team (2017). Data were tested for normal distribution using the Shapiro-Wilk test. MannWhitney test was used to compare the differences between groups. The probability function of chromosomal abnormalities depending on the values of elements in AF was analyzed. The symbol p0 indicates the probability of chromosomal abnormalities for various values of the analyzed elements. Principal component analysis (PCA) was used to analyze correlations in the data set. The correlations between elements in the group with abnormalities and in the healthy group were analyzed using Spearman's rank-order correlation. The level of statistical significance was set at $p<0.05$.

\section{Results}

Characteristics of the participants were shown in Table 1. The study population consisted of a total of 156 pregnant women with a median age of 32 years (range, 20-43 years) and pregnancy week 17 (1522 weeks). The results of the karyotype analysis indicated $49 \%$ male and $51 \%$ female fetuses. 
Chromosomal abnormalities were found in 19 fetuses (over $12 \%$ of the total population) and it was mainly trisomy $21(\mathrm{~N}=11)$, trisomy $18(\mathrm{~N}=2)$, triploidy $(\mathrm{N}=2)$ and other chromosomal aberrations. In Table 2, the content of essential and toxic elements in amniotic fluid in the total population was shown. Following genetic analysis, the samples were divided into two groups: control group (C) $(N=137)$ with normal karyotype and study group (A) ( $N=19)$ with chromosomal abnormalities. Women in the study group were significantly older than women in the control group (Table 3). The comparison of analysed elements in AF indicated a significant decrease of $\mathrm{Mn}$ concentration in the group with chromosomal abnormalities compared to the group with normal karyotype (Figs. 1 and 2).

Table 1

Characteristic of the total population (mean $\pm \mathrm{SD} /$ median/min-max)

\begin{tabular}{|ll|}
\hline Parameters & Value \\
\hline $\mathrm{N}$ & 156 \\
\hline Age (years) & $32.14 \pm 5.42 / 32 / 20-43$ \\
\hline Week of gestation & $19.24 \pm 4.94 / 17 / 12-40$ \\
\hline Gender F/M & $80 / 76$ \\
\hline Chromosome abnormalities (N): & 19 \\
\hline Trisomy + 18 & 2 \\
\hline Trisomy + 21 & 11 \\
\hline Triploidy & 2 \\
\hline mos 47,XY,+5.nuc ish 5q21(EGR-1X3)[24/64] & 2 \\
\hline $46, X X, d e l(4)(p 16.1)$ & 2 \\
\hline
\end{tabular}


Table 2

Concentration of elements in amniotic fluid in total population (mean $\pm \mathrm{SD} /$ median/min-max)

\begin{tabular}{|ll|}
\hline Element & Concentration \\
\hline $\mathrm{Li}(\mu \mathrm{g} / \mathrm{L})$ & $2.68 \pm 1.05 / 2.42 / 1.19-7.87$ \\
$\mathrm{Mg}(\mathrm{mg} / \mathrm{L})$ & $13.52 \pm 1.95 / 13.51 / 6.99-19.34$ \\
\hline $\mathrm{Al}(\mu \mathrm{g} / \mathrm{L})$ & $106.33 \pm 225.18 / 43.81 / 4.9-1972.86$ \\
\hline $\mathrm{Ca}(\mathrm{mg} / \mathrm{L})$ & $55.75 \pm 12.77 / 56.21 / 14.37-82.60$ \\
\hline $\mathrm{Cr}(\mu \mathrm{g} / \mathrm{L})$ & $0.83 \pm 1.27 / 0.50 / 0.2-9.93$ \\
$\mathrm{Mn}(\mu \mathrm{g} / \mathrm{L})$ & $1.60 \pm 1.37 / 1.26 / 0.26-9.80$ \\
\hline $\mathrm{Fe}(\mu \mathrm{g} / \mathrm{L})$ & $330.83 \pm 256.22 / 279.30 / 30.45-2369.36$ \\
\hline $\mathrm{Co}(\mu \mathrm{g} / \mathrm{L})$ & $0.10 \pm 0.06 / 0.08 / 0.02-0.39$ \\
\hline $\mathrm{Ni}(\mu \mathrm{g} / \mathrm{L})$ & $1.53 \pm 1.43 / 1.19 / 0.24-9.90$ \\
\hline $\mathrm{Cu}(\mu \mathrm{g} / \mathrm{L})$ & $138.93 \pm 71.35 / 127.99 / 18.75-726.30$ \\
\hline $\mathrm{As}(\mu \mathrm{g} / \mathrm{L})$ & $0.29 \pm 0.25 / 0.20 / 0.12-1.25$ \\
\hline $\mathrm{Se}(\mu \mathrm{g} / \mathrm{L})$ & $9.24 \pm 3.96 / 8.94 / 4-39.24$ \\
\hline $\mathrm{Sr}(\mu \mathrm{g} / \mathrm{L})$ & $18.91 \pm 15.26 / 15.24 / 2.70-124.56$ \\
\hline $\mathrm{Cd}(\mu \mathrm{g} / \mathrm{L})$ & $0.04 \pm 0.08 / 0.02 / 0.01-0.79$ \\
\hline
\end{tabular}

Table 3

Age and week of gestation in group with normal karyotype and in chromosomal abnormalities group (mean $\pm \mathrm{SD} /$ median/min-max).

\begin{tabular}{|lll|}
\hline Parameters & Normal karyotype & Chromosomal abnormalities \\
\hline $\mathrm{N}$ & 137 & 19 \\
\hline Age (years) & $31.72 \pm 5.42 / 32 / 20-43^{\mathrm{a}}$ & $35.21 \pm 4.50 / 36 / 24-43^{\mathrm{b}}$ \\
\hline Week of gestation & $18.85 \pm 4.38 / 17 / 13-32$ & $22.16 \pm 7.56 / 22 / 12-40$ \\
\hline a,b - significant differences; Mann-Whitney test; $\mathrm{p}=0.006$ \\
\hline
\end{tabular}

The probability function of chromosomal anomalies in fetuses depending on the values of elements in AF was analysed (Fig. 3). It was found that the probability of chromosomal abnormalities increased with the age of the pregnant woman. The age over 40 years was associated with more than $20 \%$ probability of the occurrence of chromosomal aberrations. A manganese level below $1 \mu \mathrm{gL}^{-1}$ was related with nearly $15 \%$ probability of the occurrence of anomalies. Moreover, the probability of abnormal karyotype 
increased with an increase in iron concentration in AF. An iron level of nearly $1500 \mu \mathrm{gL}^{-1}$ was associated with over $40 \%$ probability of the occurrence of chromosomal anomalies.

Figure 4 shows principal component analysis chart. It was observed that the first two principal components explain nearly $23.3 \%$ of the total variance and our PCA chart can only be a visualization of the correlation tendencies of the variables under consideration. It should be mentioned that each of the principal components is a linear combination of all the output variables. Principal component analysis (PCA) was based on all the analyzed variables simultaneously.Nevertheless, some trends can be observed.

It was found that $\mathrm{Cu}$ and Fe levels in $\mathrm{AF}$ in the total population were correlated (arrows are close to one another and point in the same direction). In both groups, these elements were significantly correlated (group $A, R=0.84$ and group $C, R=0.34$ ) (Figs. 5 and 6 ). Al and Ni levels in $A F$ are positively correlated (their arrowheads point in the same direction). Figures 5 and 6 show a significant correlation between these two toxic elements (in group $A, R=0.63$, in group $C, R=0.45$ ). Moreover, a high positive correlation between $\mathrm{Ca}$ and $\mathrm{Mg}$ and also between $\mathrm{Ca}$ and $\mathrm{Sr}$ and between $\mathrm{Cd}$ and $\mathrm{As}$ in $\mathrm{AF}$ was found in the total population and in both studied groups (Fig. 4-6).

\section{Discussion}

In this study, we determined the association of essential and toxic elements with chromosomal abnormalities in human amniotic fluid in fetuses of the white/Caucasian population in the second trimester of pregnancy. Based on the results, low concentrations of manganese and high concentrations of iron were associated with ahigher occurrence of chromosomal abnormalities (predominantly of trisomy 21, Down's Syndrome). To the best of our knowledge, this is the first study of Polish pregnant women to demonstrate such a relationship.

This study showed that the presence of chromosomal aberration in fetuses increased with a low level of $\mathrm{Mn}$ in AF. Mn plays a crucial role in maternal health and early pregnancy development. Manganese acts by activating certain enzymes and through Mn-dependent metalloenzymes, such as manganese superoxide dismutase, that are needed for proper reproduction and protection against free radicals. In animal and human studies, it was found that a low level of maternal manganese is associated with reduced reproductive function, impaired fetal growth and poor perinatal outcomes. On the other hand, it is known that excess manganese is a potent neurotoxin and may induce adverse neurological, reproductive and respiratory effects. Little is known about the relation between $\mathrm{Mn}$ status and chromosomal abnormalities $^{12}$. the mitochondrial oxidative phosphorylation pathway is a major source of free radicals, and MnSOD plays a crucial role in protection against these radicals and maintains the oxidant/antioxidant balance. A decrease in Mn content caused a low activity of MnSOD and disrupted this balance ${ }^{13}$. It was found that oxidative stress increases in amniotic fluid in Down's Syndrome ${ }^{13,14}$. 
It is also known that excess iron is a major cause of oxidative stress because Fe ions can generate free radicals in the Fenton reaction. Hattori et al. ${ }^{15}$ found that the content of catalytic Fe(II) in amniotic fluid inabnormal pregnancies (also involving trisomy 21 and trisomy 18) was significantly higher than in normal pregnancies. These authors concluded that catalytic iron in AF may be one of the markers of abnormal pregnancy. Our study confirms such a relationship and indicates that high iron levels in AF increased the probability of fetal abnormalities, mainly Down's Syndrome and trisomy 18 . We speculate that increased iron in AF may be swallowed by the trisomic fetus and higher Fe concentrations in its body with chromosomal abnormalities accelerate oxidative stress and progressive oxidative damage of its internal organs. We also noticed that elevated concentration of iron in AF was present not only in DS cases. Extremely high levels of iron (over $1600 \mathrm{ug} / \mathrm{L}$ ) were found in AF with triploidy.

Other trisomies are also associated with increased oxidative stress, inflammation and nervous system disorders. However, most literature reports about the relationship between minerals and chromosomal abnormalities focus on Down's Syndrome. Significantly higher ferritin levels and markedly lower transferrin concentrations and TIBC levels were found in adults (18-35 years) with DS compared to healthy individuals ${ }^{16}$.

It seems that increased oxidative stress observed in people with DS may begin in utero and might play a crucial role in phenotypic traits. Moreover, Down Syndrome and many other chromosomal abnormalities are associated with metabolic diseases such as obesity, insulin resistance, lipid disorders with a great role of oxidative stress in etiopathogenesis ${ }^{7,17}$. Therefore, based on the obtained results we can assume that increased oxidative stress linked to high Fe and low Mn concentrations in AF in early development may contribute to increased oxidative stress, brain disorders and other metabolic disorders in the later life of people with aneuploidies. It seems that a treatment strategy involving lowering oxidative stress by regulation of element status in AF may potentially protect against the disorders that are observed in people with chromosomal abnormalities.

The relationship of chromosomal aberration with lower Mn concentration and higher iron level in AF may also be associated with the interaction between Fe and $\mathrm{Mn}$ on the transport level. Divalent metal transporter 1 (DMT1) is the primary non-heme iron transporter in the intestine to transport iron but also other elements, including manganese. DMT 1 is found not only in the intestine but also in other tissues, also in the placenta, and the transport of excess iron via this transporter decreases the transport of manganese in tissues ${ }^{12}$.

The concentration of elements in AF may be affected by endogenous and exogenous factors. The correlation between essential elements observed between iron and copper levels as well as between calcium and magnesium levels indicate the transport and metabolic relationships between these elements. It is known that systemic copper deficiency generates cellular iron deficiency. These two essential elements participate in single-electron transfer reactions and owing to this capacity, they also may generate free radicals and increase oxidative status in the body ${ }^{18}$. As mentioned above, an association between iron excess and neurodegenerative diseases including Down's Syndrome was 
found ${ }^{15}$. In this study, we observed higher iron levels in AF in pregnancy with chromosomal disorders. In a mouse model of Down's Syndrome it was observed that copper accumulation in the brain increased oxidative stress $^{19}$. A change in the homeostasis of copper in the brain is also suggested in Alzheimer's disease $^{8}$. In the human body, magnesium and calcium metabolism are closely related. There are relations between the intestinal absorption and renal excretion of these two elements ${ }^{20}$. Positive correlation between toxic elements, e.g. Al and $\mathrm{Ni}$, observed in our study may be the consequence of exposure to environmental concomitants ${ }^{21}$. Correlation index values are higher for AF with chromosomal abnormalities which may be related to the exposure of pregnant women in this group to different toxic elements during pregnancy. It was found that toxic elements such as $\mathrm{Cd}, \mathrm{Ni}$ and $\mathrm{Al}$ are adversely related to chronic morbidity ${ }^{21,22}$. Results of the study by McLachlan et al. ${ }^{23}$ suggest that aluminium accumulation in the brains of patients with Alzheimer's disease and Down's Syndrome may contribute to the neuropathology of those neurological diseases.

It is known that advanced age in women increases the risks of infertility and aneuploidy in the offspring, most predominantly Down's syndrome (trisomy 21), Edwards' syndrome (trisomy 18), and Turner's syndrome (monosomy $\mathrm{X}$ ),. The postulated mechanism is connected with increased oxidative stress which may alter gene expression. Follicular aging can lead to mitochondrial dysfunction and hence disturb MnSOD activation. Chromosome segregation errors occur in aged oocytes during meiosis, which can result in aneuploidy and poor oocyte quality ${ }^{24}$.

This study has some limitations. First of all, our study group with chromosomal abnormalities was relatively small and rather heterogeneous with different chromosomal abnormalities (nearly $60 \%$ were cases with DS). Furthermore, in the present study, we did not determine nutritional and environmental factors that may affect the concentration of elements in AF. We also did not include other maternal parameters such as smoking, body mass, education and socioeconomic status that may influence the content of elements in AF.

\section{Conclusions}

Low manganese concentration and high iron level in amniotic fluid in the second trimester of pregnancy are associated with a higher incidence of chromosomal abnormalities in human fetuses.

\section{Declarations}

\section{Funding source}

This work was supported by the statutory research in Poznan University of Life Sciences (No. 506-78600-03) and it was partially funded by a grant from the Rector of the Poznań University of Technology (grant number 0213/SIGR/2154). 
The funding source had no involvement in study design; in the collection, analysis and interpretation of data; in the writing of the report; nor in the decision to submit the article for publication.

\section{Competing interests}

The authors declare no competing interests.

\section{Authors' contribution}

J.S.conceptualisation, methodology, writing-original draft and editing, supervision, J.P. sample collection, R.K. sample collection and draft editing, E.B. statistical analysis, MP and BN karyotype analysis, AS and DB elements analysis.

\section{Data availability}

The datasets generated during and/or analysed during the current study are available from the corresponding author on reasonable request.

\section{References}

1. Silberstein, T. et al. Elements in maternal blood and amniotic fluid determined by ICP-MS. J. Matern. Neonatal Med, 28, 88-92 https://doi.org/10.3109/14767058.2014.905907 (2015).

2. Suliburska, J. et al. Concentrations of Mineral in Amniotic Fluid and Their Relations to Selected Maternal and Fetal Parameters. Biol. Trace Elem. Res, 172, https://doi.org/10.1007/s12011-0150557-3 (2016).

3. Saghazadeh, A. et al. Systematic review and meta-analysis shows a specific micronutrient profile in people with Down Syndrome: Lower blood calcium, selenium and zinc, higher red blood cell copper and zinc, and higher salivary calcium and sodium. PLoS One, 12, 1-20 https://doi.org/10.1371/journal.pone.0175437 (2017).

4. Raha, A. A. et al. Hepcidin Increases Cytokines in Alzheimer's Disease and Down's Syndrome Dementia: Implication of Impaired Iron Homeostasis in Neuroinflammation. Front. Aging. Neurosci, 13, 1-17 https://doi.org/10.3389/fnagi.2021.653591 (2021).

5. Mittal, S., Boan, A. D., Pereira-Smith, S. \& LaRosa, A. Screening for Anemia in Children with Down Syndrome. J. Dev. Behav. Pediatr, 41, 141-144 https://doi.org/10.1097/DBP.0000000000000731 (2020).

6. Verrotti, A., Carelli, A., Di Genova, L. \& Striano, P. Epilepsy and chromosome 18 abnormalities: A review. Seizure, 32, 78-83 https://doi.org/10.1016/j.seizure.2015.09.013 (2015). 
7. Dierssen, M. et al. Down Syndrome Is a Metabolic Disease: Altered Insulin Signaling Mediates Peripheral and Brain Dysfunctions. Front. Neurosci, 14, 1-16 https://doi.org/10.3389/fnins.2020.00670 (2020).

8. Malakooti, N., Pritchard, M. A., Adlard, P. A. \& Finkelstein, D. I. Role of metal ions in the cognitive decline of down syndrome. Front. Aging. Neurosci, 6, 1-6 https://doi.org/10.3389/fnagi.2014.00136 (2014).

9. Karri, V., Schuhmacher, M. \& Kumar, V. Heavy metals ( $\mathrm{Pb}, \mathrm{Cd}$, As and MeHg) as risk factors for cognitive dysfunction: A general review of metal mixture mechanism in brain. Environ. Toxicol. Pharmacol, 48, 203-213 https://doi.org/10.1016/j.etap.2016.09.016 (2016).

10. Kocylowski, R. et al. Associations between the level of trace elements and minerals and folate in maternal serum and amniotic fluid and congenital abnormalities. Nutrients, 11, 1-12 https://doi.org/10.3390/nu11020328 (2019).

11. Markiewicz, B. et al. Multielemental analysis of 18 essential and toxic elements in amniotic fluid samples by ICP-MS: Full procedure validation and estimation of measurement uncertainty., 174, https://doi.org/10.1016/j.talanta.2017.05.078 (2017).

12. Wood, R. J. Manganese and birth outcome. Nutr. Rev, 67, 416-420 https://doi.org/10.1111/j.17534887.2009.00214.x (2009).

13. Barone, E. et al. Disturbance of redox homeostasis in Down Syndrome: Role of iron dysmetabolism. Free Radic. Biol. Med, 114, 84-93 https://doi.org/10.1016/j.freeradbiomed.2017.07.009 (2018).

14. Garcez, M. E., Peres, W. \& Salvador, M. Oxidative stress and hematologic and biochemical parameters in individuals with down syndrome. Mayo Clin. Proc. 80, 1607-1611. https://doi.org/10.4065/80.12.1607 (2005).

15. Hattori, Y. et al. Catalytic ferrous iron in amniotic fluid as a predictive marker of human maternal-fetal disorders. J. Clin. Biochem. Nutr, 56, 57-63 https://doi.org/10.3164/jcbn.14-82 (2015).

16. Manna, C. et al. Increased non-protein bound iron in Down syndrome: contribution to lipid peroxidation and cognitive decline. Free Radic. Res, 50, 1422-1431 https://doi.org/10.1080/10715762.2016.1253833 (2016).

17. Estrada, J. C. et al. Human mesenchymal stem cell-replicative senescence and oxidative stress are closely linked to aneuploidy. Cell Death Dis, 4, https://doi.org/10.1038/cddis.2013.211 (2013).

18. Arredondo, M. \& Núñez, M. T. Iron and copper metabolism. Mol. Aspects. Med, 26, 313-327 https://doi.org/10.1016/j.mam.2005.07.010 (2005).

19. Ishihara, K. et al. Copper accumulation in the brain causes the elevation of oxidative stress and less anxious behavior in Ts1Cje mice, a model of Down syndrome. Free Radic. Biol. Med, 134, 248-259 https://doi.org/10.1016/j.freeradbiomed.2019.01.015 (2019).

20. Grossi, E. et al. Serum magnesium and calcium levels in infertile women during a cycle of reproductive assistance. Magnes Res, 30, 35-41 https://doi.org/10.1684/mrh.2017.0421 (2017).

21. Karakis, l. et al. Exposure to metals and morbidity at eight years follow-up in women of childbearing age. Sci. Rep, 11, 1-9 https://doi.org/10.1038/s41598-021-90904-1 (2021). 
22. Röllin, H. B. et al. In utero exposure to aluminium and other neurotoxic elements in urban coastal south african women at delivery: An emerging concern. Int. J. Environ. Res. Public Health, 17, 1-17 https://doi.org/10.3390/ijerph17051724 (2020).

23. McLachlan, D. R. et al. Aluminum in Neurological and Neurodegenerative Disease. Mol. Neurobiol, 56, 1531-1538 https://doi.org/10.1007/s12035-018-1441-x (2019).

24. Mikwar, M., MacFarlane, A. J. \& Marchetti, F. Mechanisms of oocyte aneuploidy associated with advanced maternal age. Mutat. Res. - Rev. Mutat. Res, 785, 108320 https://doi.org/10.1016/j.mrrev.2020.108320 (2020).

\section{Figures}
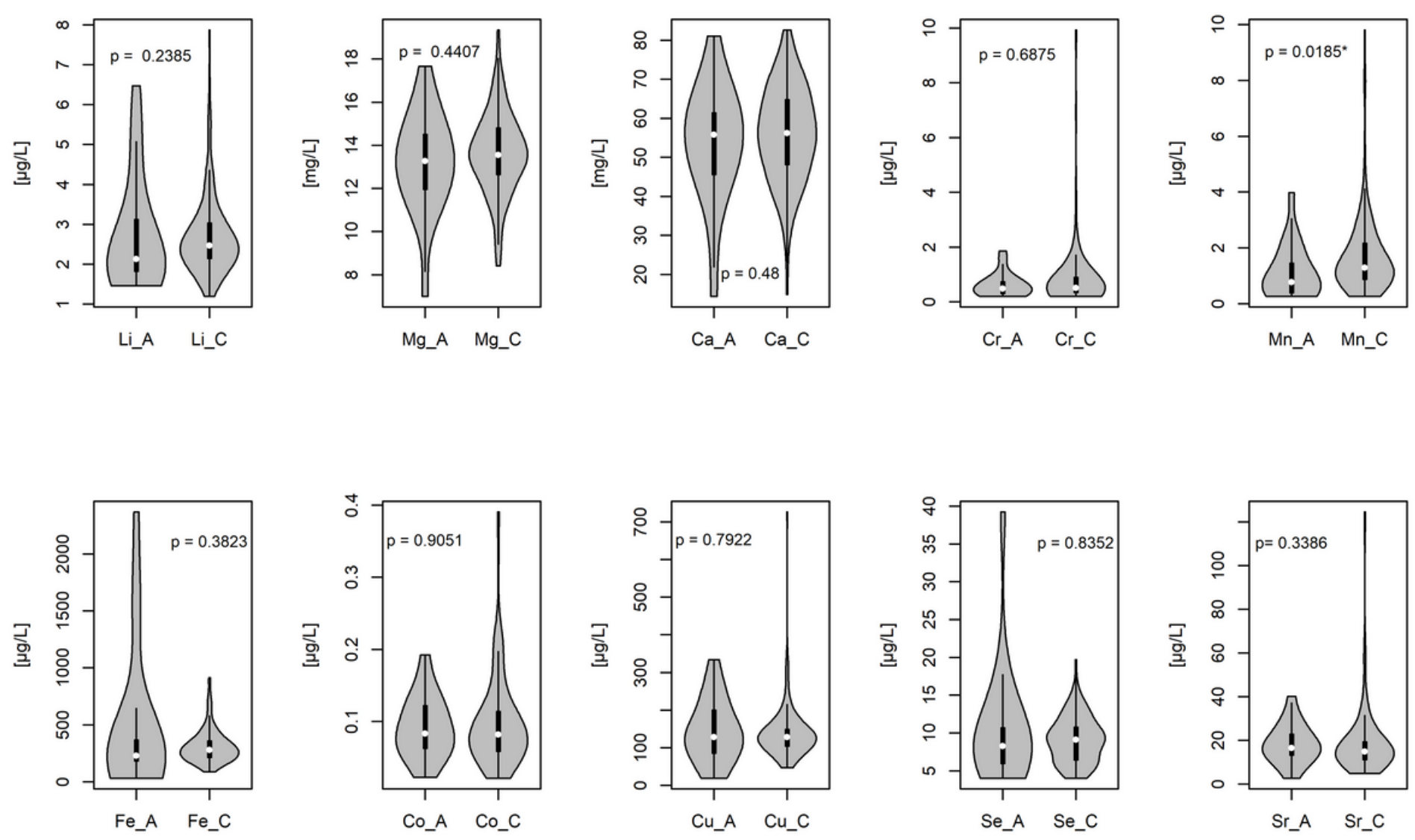

Figure 1

The concentration of essential elements in amniotic fluid; C- group with normal karyotype, group A-group with chromosomal abnormalities group; p-significance level. 

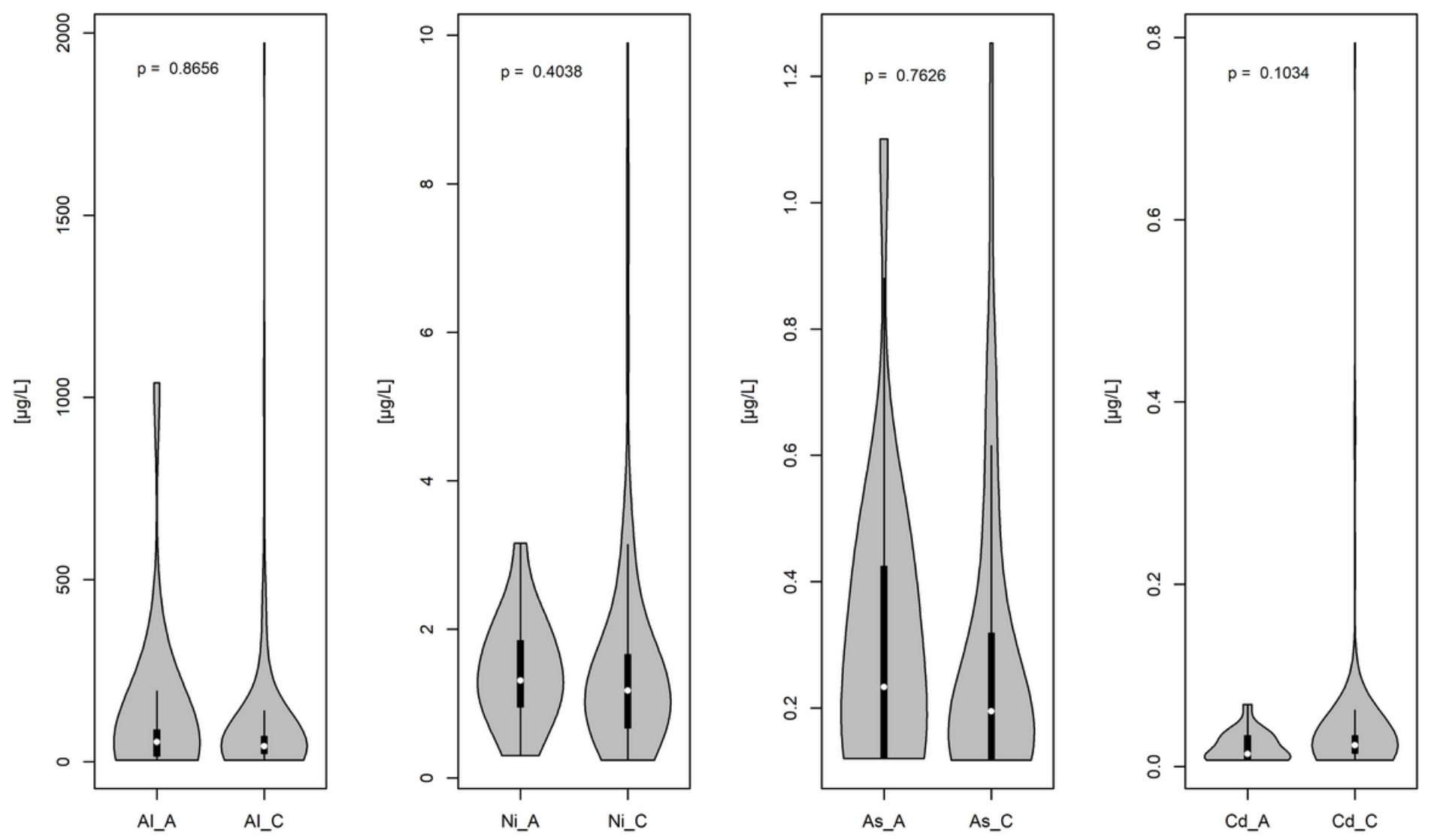

Figure 2

The concentration of toxic elements in amniotic fluid; C- group with normal karyotype, group A- group with chromosomal abnormalities group; $\mathrm{p}$-significance level. 

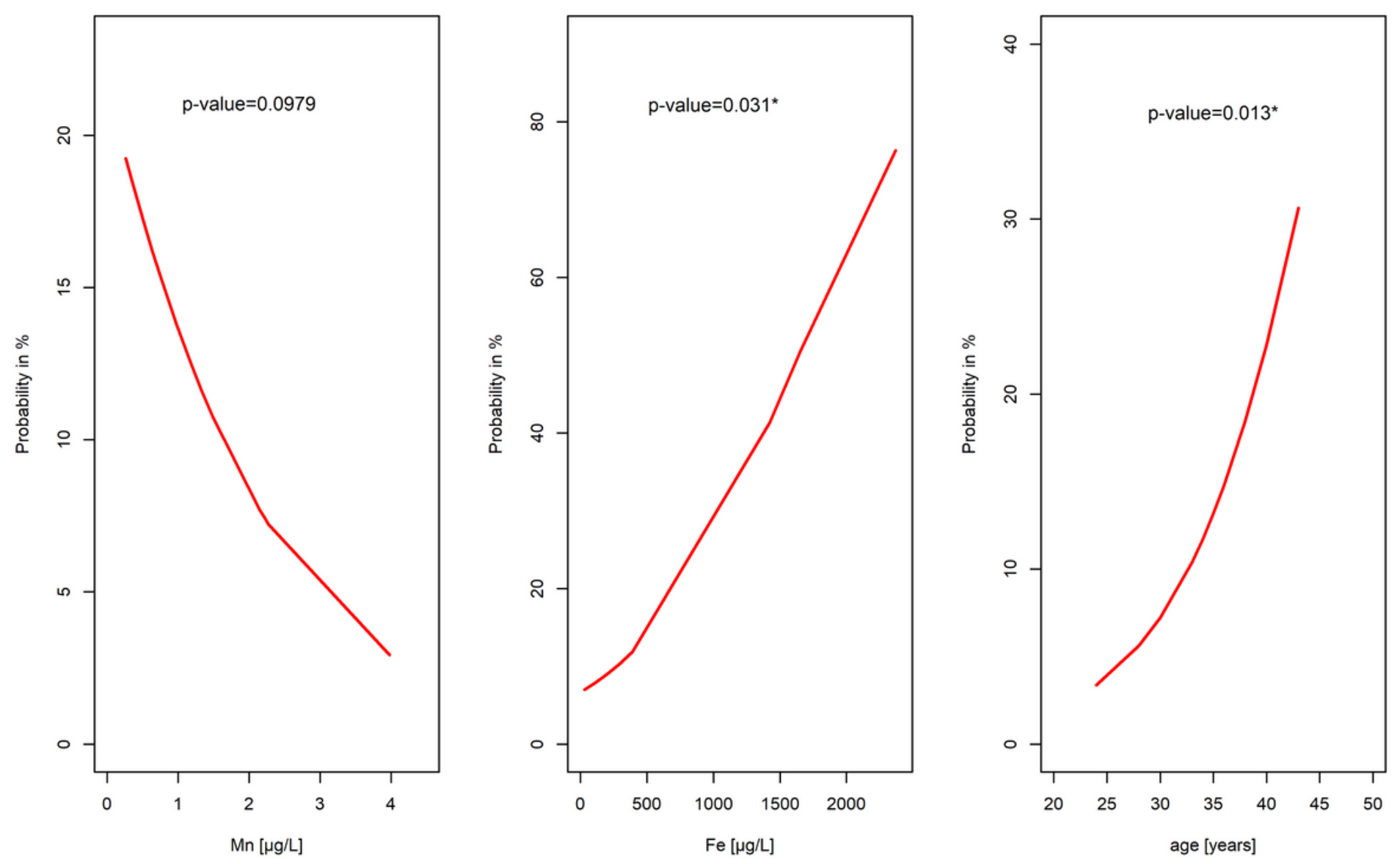

Figure 3

The probability of chromosomal abnormalities with the age and the concentration of $\mathrm{Mn}$ and $\mathrm{Fe}$ in amniotic fluid. 
PCA graph of variables

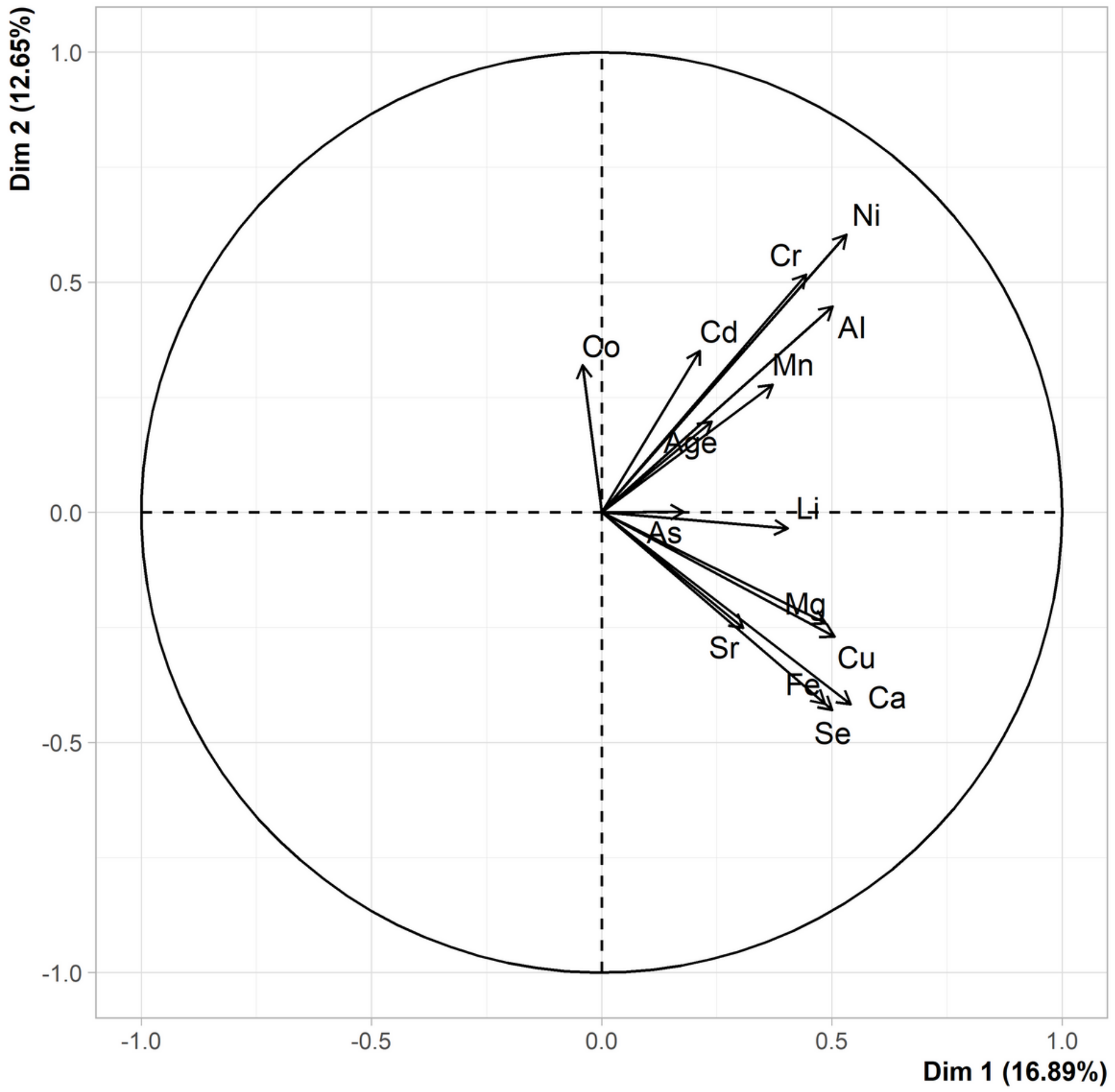

Figure 4

Principal component analysis of parameters. 


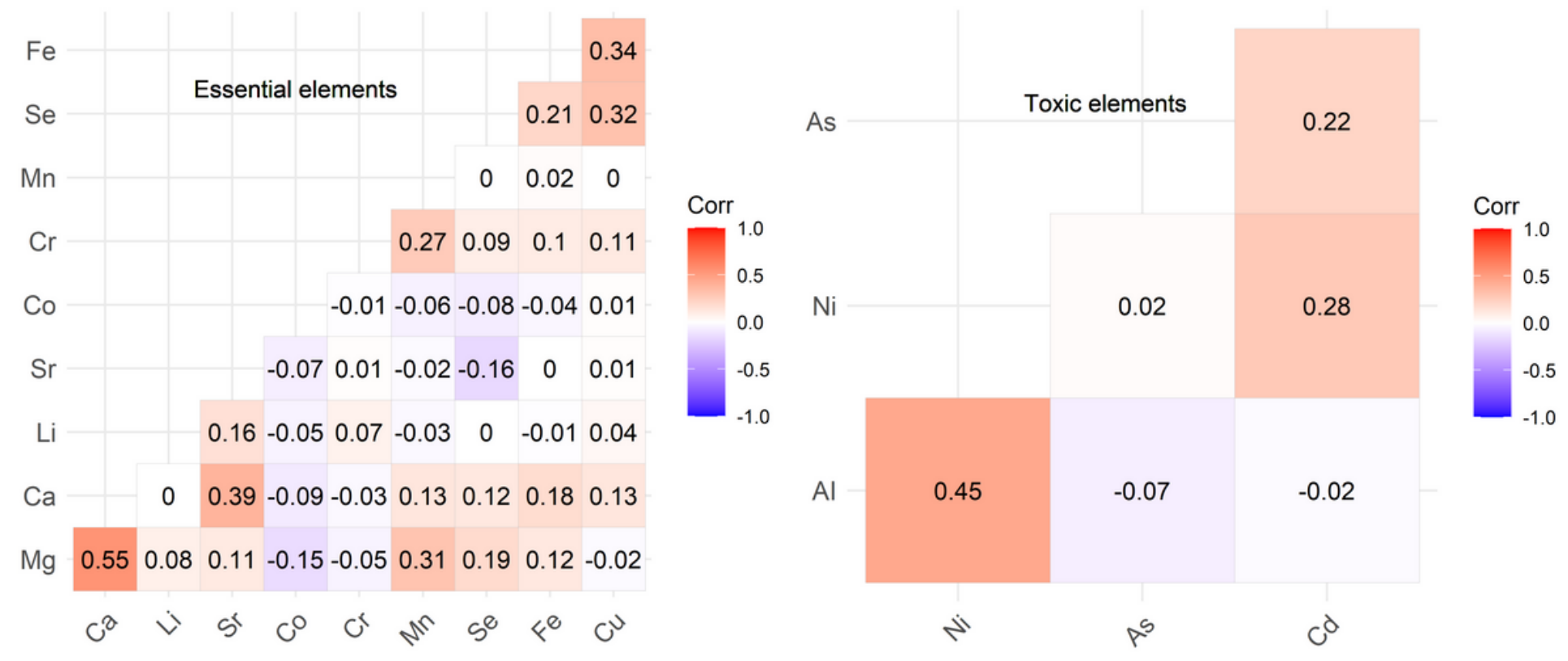

Figure 5

Correlation between essential and toxic elements in amniotic fluid of group with normal karyotype.

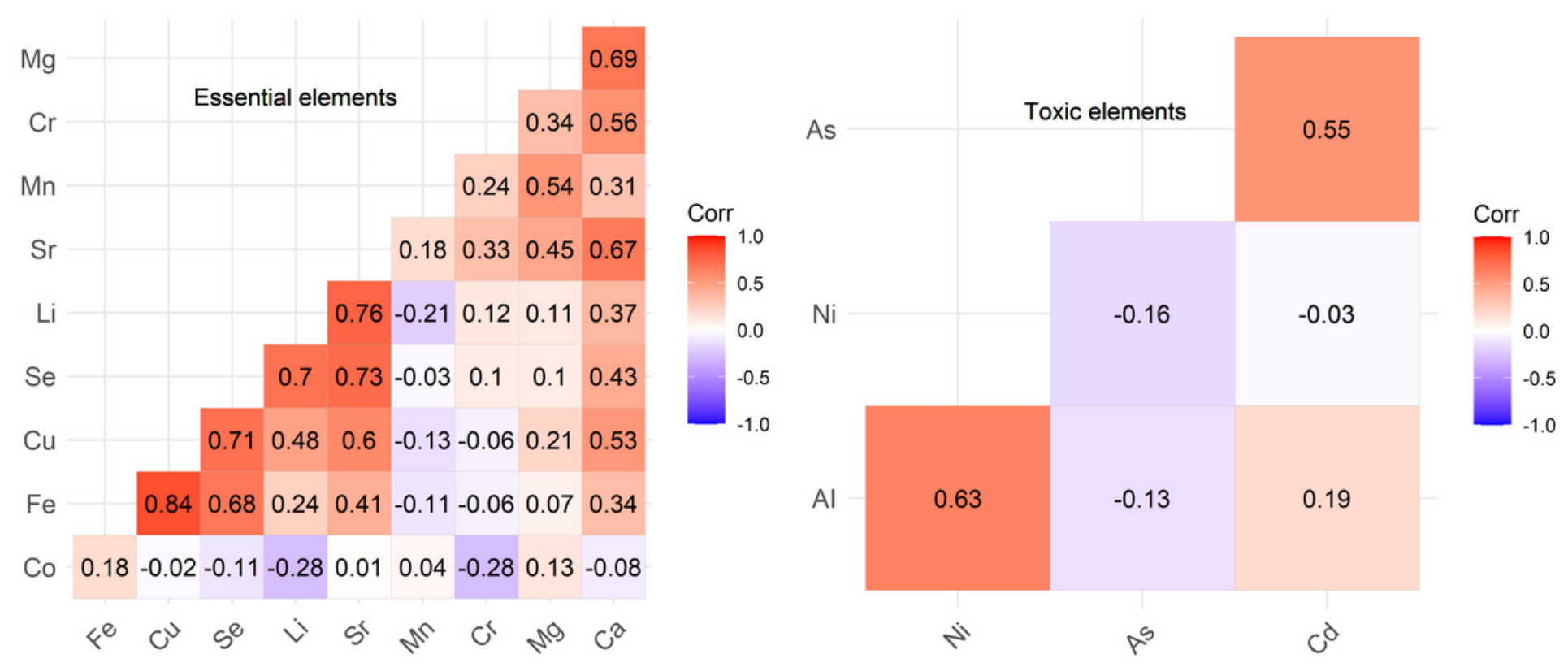

\section{Figure 6}

Correlation between essential and toxic elements in amniotic fluid of group with chromosomal abnormalities. 